\title{
A Concept of the Robotoid Manager with AR
}

\author{
Thomas Bock, Shigeki Ashida \\ The Chair for Realization and Informatics of Construction \\ The Faculty of Architecture, Technical University of Munich \\ Arcisstr. 2180333 Munich, Germany, Tel. +49-89-289-22100, Fax. +49-89-289-22102 \\ Email: Thomas.bock@bri.ar.tum.de : ashida@bri.ar.tum.de
}

\begin{abstract}
For efficiency, construction managers take care of several job sites simultaneously. In order to be omnipresent, we propose a notification of ubiquitous construction management. Here we try to adopt the system, so named the Robotoid Manager, with;

AR system with mobile computers

Message board information system

Multi schedule optimizing

Progress monitoring and schedule modification

In addition, we have taken an embedded system into account for a fast and easy recognition of real situation. Total efficiency of management of several sites is improved.

KEYWORDS: Ubiquitous management, AR (Augmented reality), Message board, Schedule optimizing, Schedule modification, Embedded system, RFID (Radio Frequency Identification)
\end{abstract}

\section{INTRODUCTION}

For efficiency, construction managers recently take care of several sites simultaneously in many cases. In order to be omnipresent, we propose a notification of ubiquitous construction management by adopting of augmented reality system with mobile computers.

It is able for a robotoid manager to reduce a time of transfer to each job-site, and to have communication better with more people who are not on the site and even not concerned directly with the construction. Just-in-time decision and fast information transmitting will be possible, because of accessing updated and large volume of data from various aspects, whenever and wherever he/she is.

There are also a lot of chances to do another task at a different situation while managing. It reduces a total required time of work.

\section{Difficulties}

\subsection{Differences of situation}

Under a ubiquitous management, the situation of managing may often be quite different from the site. There are also many other disturbances. For example, there are many people, e.g. in an office, who are not concerned on the site.

And processes on the site are not recognized directly. If the manager is always at the site, he/she can supervise all activities. Sometimes it is said that these human senses are most important to make a best decision. A robotoid manager must recover this lack of recognition as well by using additional communication devices.

\subsection{Confusion of several sites}

Different situations of each site may be sometimes not allocated to the proper site, especially caused by unexpected apprehension or unclear information. The manager must always take it into account, which information belongs to which site. Misunderstandings may sometimes result in a serious loss of efficiency. 


\section{A concept of the Robotoid Manager}

\subsection{Possibilities of AR system}

Here we can adopt an augmented reality system (shortly AR) with mobile computers for a robotoid manager. Many of these systems have been already introduced in the market; sometimes as wearable style that means it is easy to carry and use. It provides anywhere a similar vision as that on the site, and also free from a disturbance in different surrounding conditions. A robotoid manager can always wear all related data and communication tool with visual help of AR. AR can also increase efficiency of communication without oral method.

With an AR system, in addition we can take following possibilities.

- Visual comparison between a plan and a real progression.

- High visibility

Without a dead angle / Through obstacles / Inner-structure / Underground / Clear through dense dusts, darkness, mud, water etc.

- Visual help for a consideration

Situation of temperature, Humidity, air flow, direction and level of lighting, smell, sound and others

- Indication for a pointing of position and time

- Management of construction materials and parts

- Pre-check of a process and effect simulation
- Communication crossover situations

- For training

In case information should be shared by other people, it can either be projected on screen or on each person goggles. For better visibility, a larger screen is necessary, which is harder or more troublesome to carry. Then a useful indication device, for example a mobile or wearable beamer, should be the best combination.

\subsection{Message board information system}

A manager must always be aware of a present situation, which changes time to time, in order to make a just-in-time decision. Therefore it is better to use real time data and their recent previous related actions, instead of using too much stored data. Too much data require many capacity of computer server, and they are sometimes never used anymore. And also it requires for a manager complicate searching, comparing and selecting the proper data.

To get real new data while classifying, message board information system (Fig.1) is a practical method. This method means to gather opinions of all workers at each site at the real time. Due to the use of this system, we can spare sub managers on various site. An Internet message board system with a mobile communication device has been already used. So almost all worker on site can use this system easily without any complicate installation or any expensive devices.

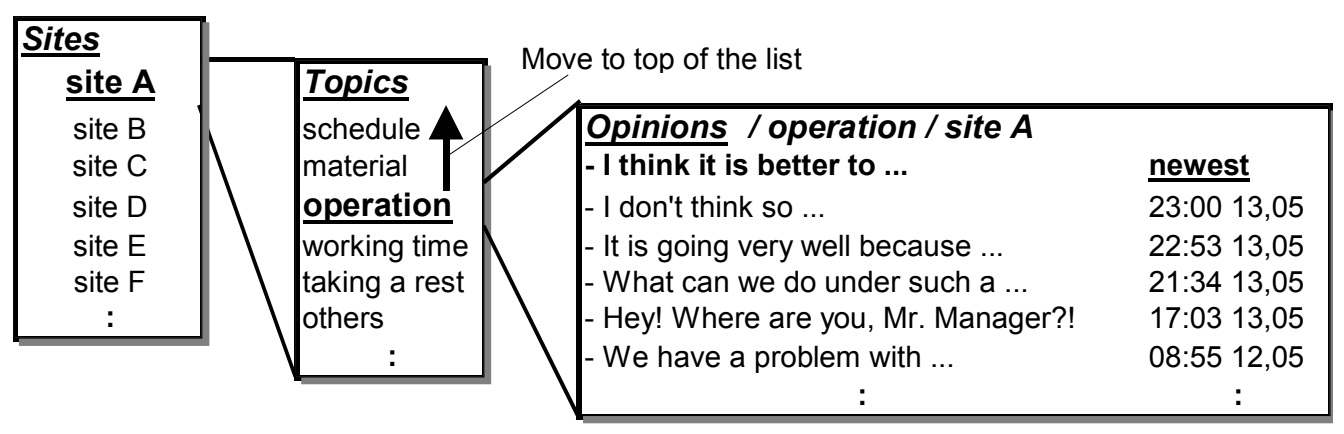

Fig.1. Message board information system 
opinions, but also informing a decision of the signal and make a better decision as soon as manager.

same timpossible.

3.3. Optimizing the combination of each other site $\mathrm{T}_{\mathrm{r}}^{\text {Sequence }}$ For a changed situation;" the "sehedule of each site Optimized $T_{r} \quad T_{f}$ must always be modified $t_{f}$ with an analysis of the While sequencesscheduderal sites are progressing in

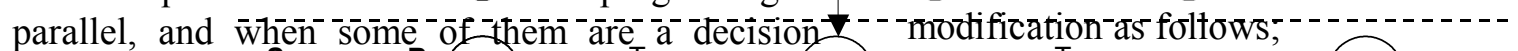

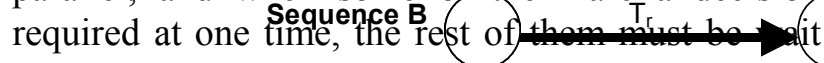
for a decision. So it is the best that there comes

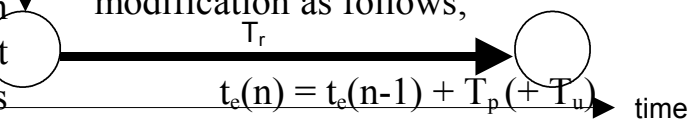

always one decision required sequence after another. A combination between Criticab sequence sequences and decision required sqquepees shopld sequence be optimized automatically with a multi schedule optimizing method.
Here, $t_{e}(n): t_{e}$ of the sequence " $n$ ", $t_{e}(n-1): t_{e}$ of the previous sequeision requiked node $\Gamma_{p}$ :a required time which is predicted from a real progress of the sequence, $\mathrm{T}_{\mathrm{u}}$ : a required time of the unscheduled sequence (if

\section{Fig.2. A concept of multi Befiesfary bptimizing}

All sequences are related each other owing to its order and required time. It is indicated as an arrow diagram. A progress is monitored and its criticality of time is considered. A flexibility of the sequence is evaluated as follows;

$$
T_{f}=\left(t_{1}-t_{e}\right)-T_{r}
$$

Here, $\quad T_{f}$ : a flexible time, $t_{1}$ : the latest time to be allowed, $t_{\mathrm{e}}$ : the earliest time to be able to start, $\mathrm{T}_{\mathrm{r}}$ : a preset required time

When $T_{\mathrm{f}}=0$, then the sequence is critical and the decision for the next sequence must be prepared before the time of

The index $t_{e}$ is always rewrote owing to a monitoring of the progress. Rewriting concept is shown in Fig.3. $T_{p}$ is predicted by comparing a progress with a preset required time. There may be also a case that an unscheduled sequence becomes necessary.

And the optimizing of a combination, which is described before, is repeated over and over automatically for all these modification to make the total efficiency higher. This system indicates all better possibilities up to this optimizing whenever a decision is required for a ubiquitous manager.

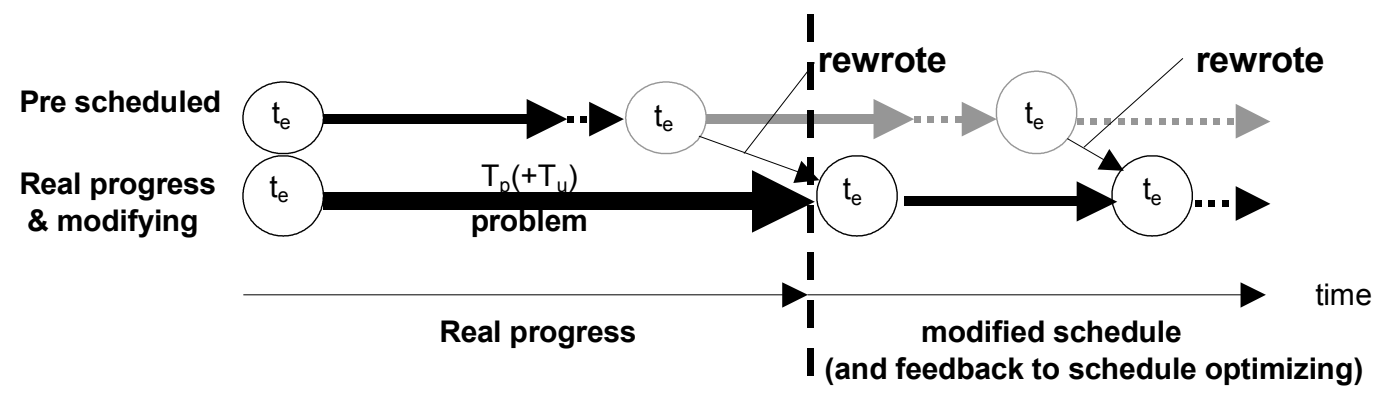

Fig. 3. Rewriting concept on the modification

\section{Situation recognizing with embedded system}

\subsection{Installation of RFID}

All decisions should be always made regarding a present requirement. Making a decision effectively 
we analyse the present situation and refer the consolidated findings in the future.

3D scanning with AR is one of the best methods to grasp the real situation. AR itself with wearable computers has also already been popular. But in the use of $\mathrm{AR}$ for $3 \mathrm{D}$ scanning, there are still some technical and practical difficulties to realize. It is sometimes expensive, inexact and time for scanning is required. Also it is still hard to carry or wear the equipment easily, for example still many devices are required as that for recognizing self position. Therefore an accepted technique method only with visual 3D scanning for every concerned site is not practice to use, at the moment.

On the other hand, RFID (Radio Frequency Identification) is one of the popular identification methods. The chip is embedded in all objects at each site containing its specific data. The scanner for this system is in most of the cases easy to carry or wear.

\subsection{Concept of recognizing}

We are here trying to adopt an embedded system with RFID and AR system with mobile computers for recognizing of sites to a robotoid manager.

Specific data of all objects at the site are preliminary registered and embedded with RFID chip. In case, more detail data must be stored, the identification is related to data bank automatically (Fig. 4).

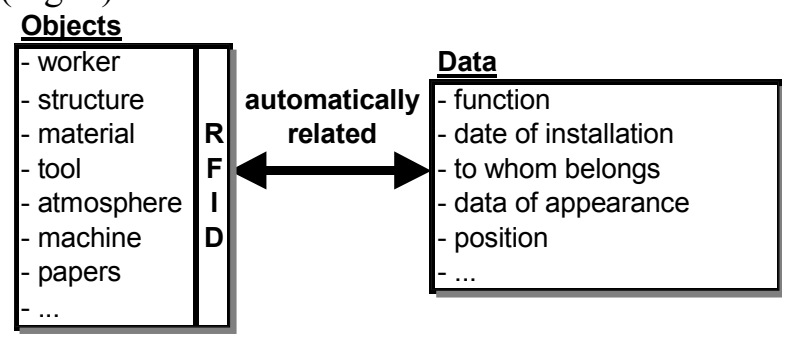

\section{Fig. 4. Embedded data}

With RFID all objects on the site in the radio effective distance are identified at the same time and grasped its specifications fast and easily. The visual situation, which means position, wearing, movement and others, is shown virtually with AR, like as Fig. 5. The manager can take also an invisible object into account. The ability of RFID for rewriting is important as well. On the view of $\mathrm{AR}$, a robotoid manager compares the real present situation with the virtual view, which made from pre registered RFID data (Fig. 5). Then the difference is rewritten on RFID system.

\section{Future work}

This trial is still just a concept. There has been no real adoption yet. We must think furthermore about the realization. The concrete systems should be created and combined at its best. And of course its efficiency must be confirmed with some case study or practical adoption.

Furthermore this method will be used in many other kind of management, for example buildings, facilities, infrastructures and others. Therefore this method should be not only used to analyse the construction site, but also included into total system of management to be used effectively. We will try to implement such a robotoid management system with AR.
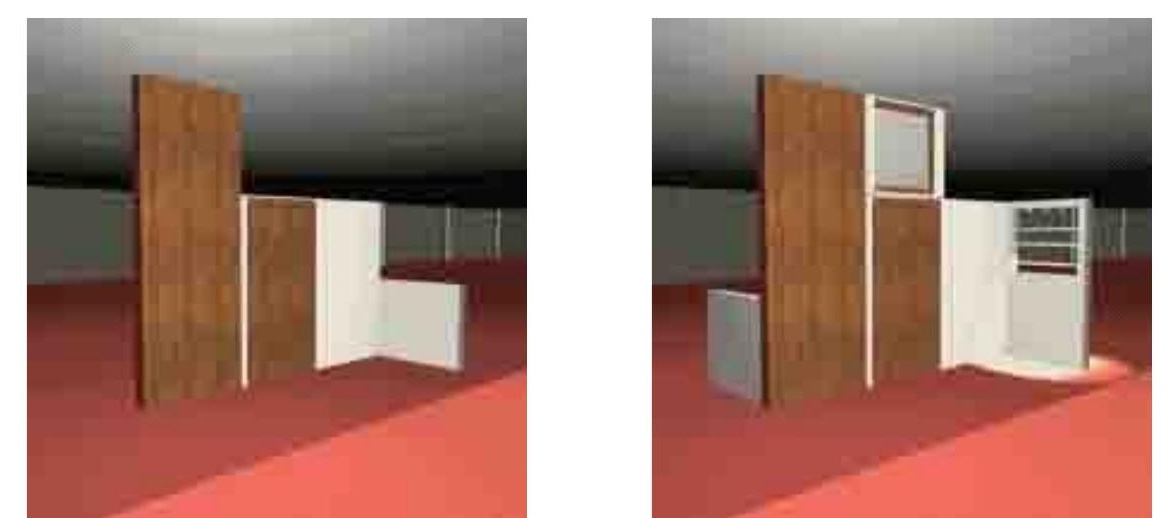

a) real situation
b) scheduled situation

Fig.5. Comparison on the view of AR 